\title{
Photonic Crystal based Amorphous Silicon Microcavity
}

\author{
Ali Serpengüzel* \\ Koç University, Microphotonics Research Laboratory, Physics Department, \\ Rumeli Feneri Yolu, Sariyer, Istanbul 34450 Turkey
}

\begin{abstract}
In this research, properties of bulk and microcavity hydrogenated amorphous silicon nitride are studied. Microcavities were realized by embedding the active hydrogenated amorphous silicon layer between two dielectric mirrors. The dielectric mirrors were realized with two distributed Bragg reflectors (DBR's). The DBR's are one dimensional photonic bandgap (PBG) materials, i.e., photonic crystals, composed of alternating layers of silicon oxide and silicon nitride. All of the layers are grown by plasma enhanced chemical vapor deposition (PECVD) on silicon substrates. The temperature dependence of the amorphous silicon photoluminescence is performed to fully characterize and optimize the material in the pursuit of obtaining novel photonic microdevices. Photonics device characterization was done by means of atomic force microscopy (AFM), scanning electron microscopy (SEM), photoluminescence, and reflectance measurements. The reflectance spectra calculations were performed using the transfer matrix method (TMM).
\end{abstract}

Keywords: Amorphous silicon, Distributed Bragg reflector, Guided waves, Fabry-Perot, Microcavity, Optoelectronics, Photoluminescence, Resonators, Spontaneous emission, Thin films.

\section{INTRODUCTION}

\subsection{Amorphous silicon}

In the optoelectronics and microelectronics industry silicon is the most widely used semiconductor, not only in its crystalline, but also in its amorphous form. Being a direct band-gap material, ${ }^{1}$ unlike crystalline silicon, ${ }^{2}$ amorphous silicon is unmatched as a photoreceptor for laser printing, for switching elements in large area liquid crystal displays, for large photovoltaic panels, and any other application that calls for a high quality semiconductor that can be processed on large areas or on curved or flexible substrates. It is generally agreed that the terms amorphous solid, non-crystalline solid, disordered solid, glass, or liquid have no precise structural meaning beyond the description that, the structure is not crystalline on any significant scale. The principal structural order present is imposed by the approximately constant separation of nearest-neighbor atoms or molecules. Until the early 1970's, amorphous silicon prepared by evaporation or sputtering was not considered as one of the valuable semiconductor materials, because of high density of electronic states in the band gap related to a large density of structural defects. ${ }^{3}$ The discovery of an amorphous silicon material prepared by the glow discharge deposition of silane, which can be doped and whose conductivity can be changed by ten orders of magnitude marked a turning point and opened a new research area. Most interest focused on hydrogenated amorphous silicon and its alloys, since hydrogen by removing dangling bonds eliminates non-radiative recombination centers, that are responsible for reduced luminescence efficiency (and reduced photovoltaic efficiency) and allows doping. ${ }^{4}$ Another advantage of the hydrogenated amorphous silicon is that, it can be deposited by plasma enhanced chemical vapor deposition (PECVD) onto almost any substrate at temperatures below $500 \mathrm{~K}$, which makes it compatible with the microelectronic technology.

\subsection{Photonic microcavities}

Photonic microcavities are currently experiencing a boom. Together with the complimentary microstructures of photonic bandgap (PBG) materials, these microresonators attract the attention of the scientists and engineers in the field of optics and photonics. As we enter the information age, the photonic microcavities is the building block of the communication revolution. There are basically two major communities, who are interested in these optical

* aserpenguzel@ku.edu.tr; phone: + 90 (212) 338-1312; fax: + 90 (212) 338-1547, http://home.ku.edu.tr/ aserpenguzel, http://microphotonics.ku.edu.tr, Koç University, Microphotonics Research Laboratory, Physics Department, Rumeli Feneri Yolu, Sariyer, Istanbul 34450 Turkey. 
microcavities. The atomic physics and condensed matter physics communities approach these optical microstructures with the scientific and basic research interest. For the physicists, the main interest in these optical microstructures is for their ability to sustain high optical fields, and therefore strongly interact with the material with which the optical microresonator is constructed from. The optoelectronics community however has a purely technological interest and approaches these optical resonators with an applied research interest. The optical communication engineers would like to manufacture high efficiency light sources such as lasers and light emitting diodes, and photodetectors for dense wavelength division multiplexing applications for optical fiber communication. From an historical perspective, we can label the birth of the first optical resonator with the invention of the first optical interference spectroscope by Charles Fabry and Alfred Perot in 1899. Another major breakthrough happened in 1946 when E. M. Purcell proposed the use of electromagnetic resonators for the manipulation of the density of states of the electromagnetic field modes. Optical resonators with dimension of the order of an optical wavelength can now be fabricated in a variety of solid state systems including semiconductors, organic materials, and glasses. ${ }^{5}$ Ideally, one can isolate a single optical mode of the optical field in a cube a half-wavelength on a side with perfectly reflecting walls. Liquid droplets, polymer spheres, and semiconductor microcavities with dielectric mirrors are examples of microcavities with which, one can approach this ideal limit, and nearly isolate a few modes of the electromagnetic spectrum from the continuum of the surrounding freespace modes. The simplest approach to fabricate an optical microcavity is to shrink the spacing between the mirrors of a Fabry-Perot resonator to a half wavelength. This structure provides a single dominant longitudinal mode, that radiates into a narrow range of angles around the cavity axis. The interaction of optically active material with isolated modes in the cavity offers interesting physical systems for basic studies and a rich variety of possible applications. Interactions between atoms and low-loss optical and microwave cavities have led to the demonstrations of cavity quantum electrodynamics effects, (QED) including coupled atom-cavity modes, quantum revivals, single-atom masers, and enhanced or inhibited spontaneous emission (SE). Microcavity resonators have the potential to provide low-cost, efficient, and high-density optoelectronic light sources over a broad range of the spectrum, from the near-infrared to well into the visible. They can be used as very efficient light emitting diodes and low threshold microlasers. ${ }^{6}$ In any macroscopic laser, SE is a major source of energy loss, speed limitation and noise. The fraction of the SE that is coupled into a single lasing mode can be increased by the enhancement and of SE by the microcavity. The efficiency emission rates, and photon statistics of light emitting diodes can all be controlled by forming microcavity resonators with sufficiently high quality factors. Control of these properties will be welcome in a number of optoelectronic applications including flat panel electroluminescent displays and optical interconnects.

\subsection{Photonic bandgap materials}

The concept of Bragg reflection was recognized by the 1915 Nobel Prize awarded jointly to W. Henry Bragg and his son W. Lawrence Bragg 'for their services in the analysis of crystal structure by means of X-rays.' The optical application of Bragg Scattering was analyzed for the first time by P. Rouard in 1937 using multiple transparent optical layers. However, these one dimensional distributed Bragg reflectors (DBR's) were used only as band reject optical filters and their potential has not been fully realized, until Eli Yablonovitch and Sajev John independently proposed in 1987 the concept of three dimensional photonic crystals or Photonic bandgap (PBG's) materials. The PBG idea is borrowed from solid state physics, where electronic bandgaps exist for an electronic crystal structure, and prevent the existence of electronic levels at certain energies. Currently, this microscopic branch of photonics and optoelectronics is experiencing a rapid and exciting development and growth. PBG materials are constructed by spatially patterning the dielectric function of a medium in a periodic way. A medium engineered in such a way exhibits photonic stop bands, i.e., PBG's, for a certain range of frequencies of the electromagnetic spectrum. A PBG structure can also be treated as a photonic crystal constructed from "photonic atoms", i.e., dielectric "photonic resonators", which possess electromagnetic resonances at discrete frequencies. Photonic atoms are the electromagnetic analogue of the real "electronic" atoms, which possess electronic resonances at discrete frequencies. The distributed Bragg reflectors used in the amorphous silicon microcavities are examples of one-dimensional PBG's.

\section{AMORPHOUS SILICON}

Interest in silicon $(\mathrm{Si})$ as a material for optoelectronics has increased recently. With modern process techniques, it will be possible to integrate lasers, photodetectors, and waveguides into optoelectronic silicon motherboards to route and modulate optical signals within such silicon motherboards. Integrated silicon optoelectronics is a rapidly developing field ${ }^{7}$. Discrete and integrated devices such as photodetectors, modulators, light emitters, resonant cavity enhanced (RCE) photodetectors, waveguides, photonic bandgap filters, optical amplifiers, optical interconnects, and 
optoelectronic integrated circuits are already being realized. However, most of these devices, with the exception of light emitters, are fabricated using crystalline silicon. Light emission requires the use of amorphous silicon, since crystalline silicon can not emit light due to its indirect bandgap. Hydrogenated amorphous silicon (a-Si:H) is already an established material in semiconductor technology ${ }^{8}$. The major application of a-Si:H is photovoltaics, color detectors, and active matrix displays. The primary attribute of the technology is its large area capability, which is unavailable with other technologies. Another advantage of a-Si:H is that, it can be deposited by plasma enhanced chemical vapor deposition (PECVD) on almost any substrate at temperatures below $500 \mathrm{~K}$, which makes it compatible with the microelectronic technology. This property justifies the interest in a-Si:H as a potential optoelectronic material. Planar waveguides are already being realized from a-Si:H ${ }^{9}$. With modern process techniques, it will be possible to integrate lasers, photodetectors, and waveguides into Si motherboards ${ }^{10,11}$ for wavelength division multiplexing (WDM) applications ${ }^{12}$.

\subsection{Luminescence of amorphous silicon}

The advantage of a-Si:H, as well as porous silicon $(\pi-\mathrm{Si})$, is that, they attract interest as a potential optical gain medium, because of their room temperature visible electroluminescence (EL) and photoluminescence (PL). Planar microcavity effects on the PL of $\pi-\mathrm{Si}^{13}$ as well as $\mathrm{Si} / \mathrm{SiO}_{\mathrm{x}}$ superlattices ${ }^{14}$ have already been reported. Recently, we have observed visible PL from a-Si:H, as well as its oxides $\left(\mathrm{a}-\mathrm{SiO}_{\mathrm{x}}: \mathrm{H}\right)$ and nitrides $\left(\mathrm{a}-\mathrm{SiN}_{\mathrm{x}}: \mathrm{H}\right)$ grown by low temperature PECVD. ${ }^{15}$ While the exact mechanism of the occurrence of the PL in bulk a-SiN $: H$ is still under discussion, the quantum confinement model is a widely accepted ${ }^{16}$ In the quantum confinement model the material consist of small a-Si clusters in a matrix of a-SiN $: \mathrm{H}$. The regions with $\mathrm{Si}-\mathrm{H}$ and $\mathrm{Si}-\mathrm{N}$, having larger energy gaps due to strong $\mathrm{Si}-\mathrm{H}$ and $\mathrm{Si}-\mathrm{N}$ bonds, isolate these a-Si clusters, and form barrier regions around them. The PL originates from the a-Si clusters. A-SiN $\mathrm{x}: \mathrm{H}$ can be grown both with and without ammonia $\left(\mathrm{NH}_{3}\right)$. The samples grown without $\mathrm{NH}_{3}$ are referred to as the $\mathrm{Si}$ rich samples. The luminescence of these samples is in the red-near-infrared part of the optical spectrum. The samples grown with $\mathrm{NH}_{3}$ and annealed at $800^{\circ} \mathrm{C}$ are referred to as the nitrogen rich samples. The luminescence of these samples is in the blue-green part of the optical spectrum ${ }^{17}$.

\subsection{Photoluminescence measurements}

The room temperature PL setup consists of a pump laser, a spectrometer, a lock-in amplifier, and a digital oscilloscope. The collected PL signal is imaged to the entrance slit of the spectrometer, whose output is fed to a sensitive photomultiplier tube (PMT). A lock-in-amplifier is providing the necessary electronic gain to the PMT output gain in phase with the laser pulse. The digital oscilloscope is used for monitoring and optimizing the PMT signal. All of the measurement and test devices are computer controlled and the data is acquired digitally. For the room temperature measurements at $300 \mathrm{~K}$ the samples are attached to a holder. For low temperature measurements the samples are placed in a closed cycle cryostat system. The closed cycle cryostat system is used to control the sample temperature from $10 \mathrm{~K}$ to $300 \mathrm{~K}$.

\subsection{Temperature dependence of the PL}

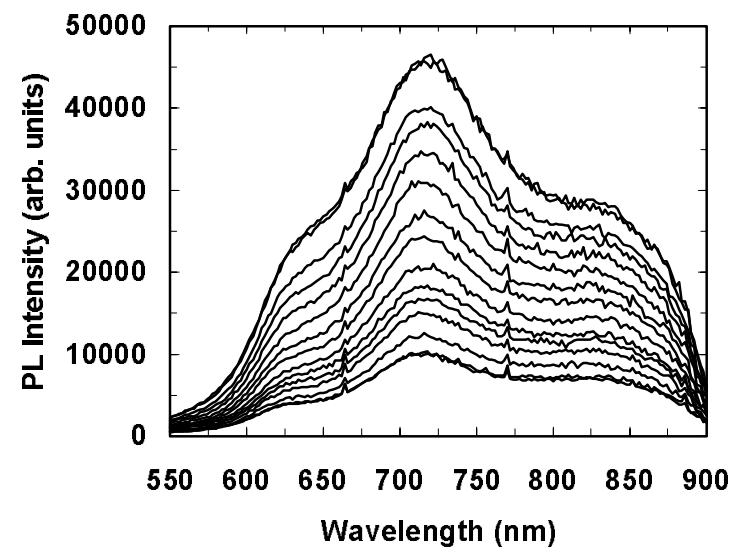

Figure 1. PL spectra of a-SiNx:H in the $12-298 \mathrm{~K}$ temperature range. 
Figure 1 shows the PL spectra of the a-SiN $\mathrm{x}: \mathrm{H}$ measured in the $550-900 \mathrm{~nm}$ wavelength and in the $12-298 \mathrm{~K}$ temperature range at a constant excitation laser intensity of $0.1 \mathrm{~W} \mathrm{~cm}^{-2}$. A broad PL band centered at $710 \mathrm{~nm}\left(E_{p}=\right.$ $1.746 \mathrm{eV}$ ) at $12 \mathrm{~K}$ is observed. The PL spectra have approximately a Gaussian lineshape modulated slightly by FabryPerot resonances. These resonances are due to the Fresnel reflections from the a-SiN $\mathrm{x}: \mathrm{H}$ film surfaces. The PL intensity decreases with increasing temperature. This feature is typical of the PL, which is due to donor-acceptor pair transitions observed in semiconductors.

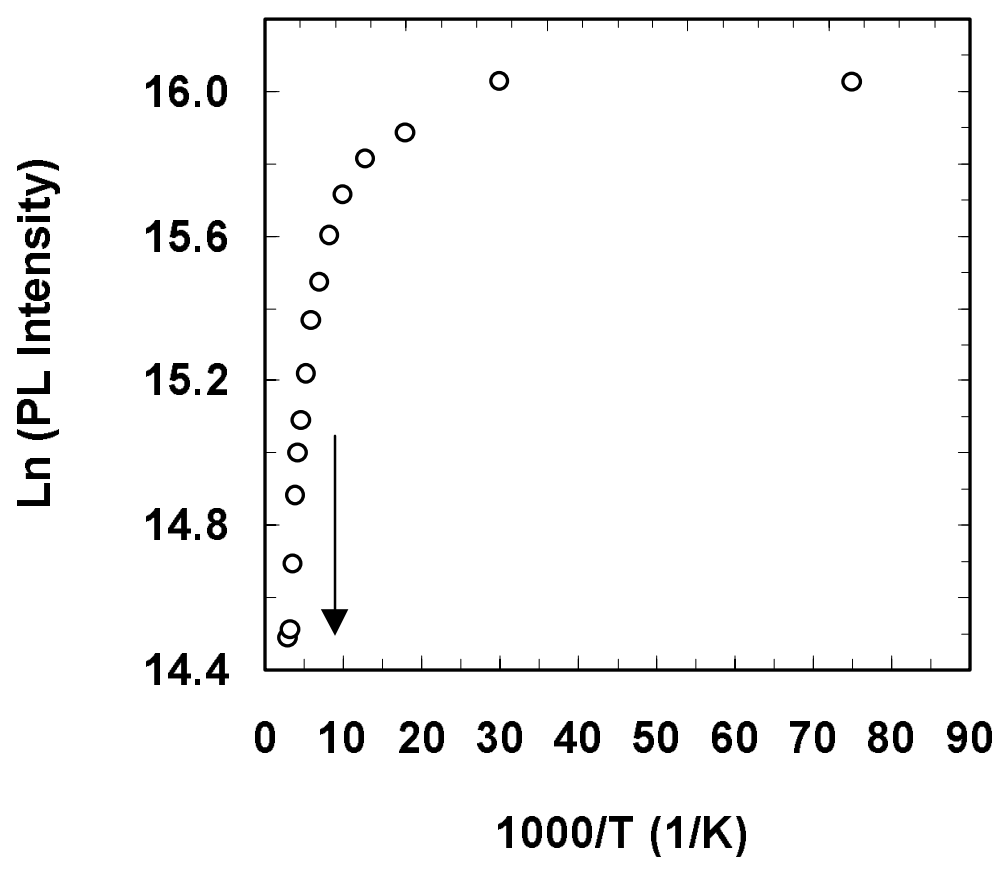

Figure 2. Temperature dependence of $\mathrm{a}-\mathrm{SiN}_{\mathrm{x}}: \mathrm{H}$ PL intensity at the emission band maximum. The arrow at $170 \mathrm{~K}$ shows the starting point of the intensive quenching.

The variation of the PL peak with respect to temperature is plotted in the figure 2 . In the 12 - $170 \mathrm{~K}$ range, the PL intensity decreases slowly. Above $170 \mathrm{~K}$, however, the PL intensity decreases at a larger rate due to a thermal quenching process. The activation energy $\Delta \mathrm{E}$ for this thermal quenching process can be derived in the $170-298 \mathrm{~K}$ temperature range using a nonlinear least squares fit to the following equation, $\mathrm{I}=\mathrm{I}_{\mathrm{O}} \exp \left(\Delta \mathrm{E} / \mathrm{k}_{\mathrm{B}} \mathrm{T}\right)$, where $\mathrm{I}$ is the PL intensity, $\mathrm{I}_{\mathrm{o}}$ a proportionality constant, and $\mathrm{k}_{\mathrm{B}}$ the Boltzmann's constant. The semilog plot of the emission band intensity as a function of the reciprocal temperature gives a straight line in the $170-298 \mathrm{~K}$ region. An activation energy of $\mathrm{E}_{\mathrm{a}}=0.027 \mathrm{eV}$ for the emission band is derived from the slope of the straight line fit. This activation energy is associated with a shallow level located at $0.027 \mathrm{eV}$ from the band. This shallow level in undoped a-SiN $\mathrm{x}: \mathrm{H}$ may be associated with the presence of defects and unintentional impurities.

\subsection{Power dependence of the PL}

Figure 3 presents the PL spectra for 10 different laser intensities at $12 \mathrm{~K}$. As the power is increased the PL intensity increases. The intensity variation of the emission band versus the excitation laser intensity at $\mathrm{T}=12 \mathrm{~K}$ is also investigated and plotted in the figure 4 . The experimental data can be fitted by a simple power law, $\mathrm{I} \propto \mathrm{L}^{\gamma}$, where I is the PL intensity, $\mathrm{L}$ the excitation laser intensity, and $\gamma$ a dimensionless exponent. It was found that, the PL intensity increases sublinearly (i.e., $\gamma=0.89$ ) with respect to the excitation laser intensity. For an excitation laser photon with an energy exceeding the band gap energy $E_{g}$, the coefficient $\gamma$ is generally $1<\gamma<2$ for the free- and bound-exciton emission, and $\gamma \leq 1$ for free-to-bound and donor-acceptor pair recombination. Thus, the obtained value of $\gamma=0.89$ confirms the assignment of the observed emission band in $\mathrm{a}-\mathrm{SiN}_{\mathrm{x}}: \mathrm{H}$ is due to donor-acceptor pair recombination. 


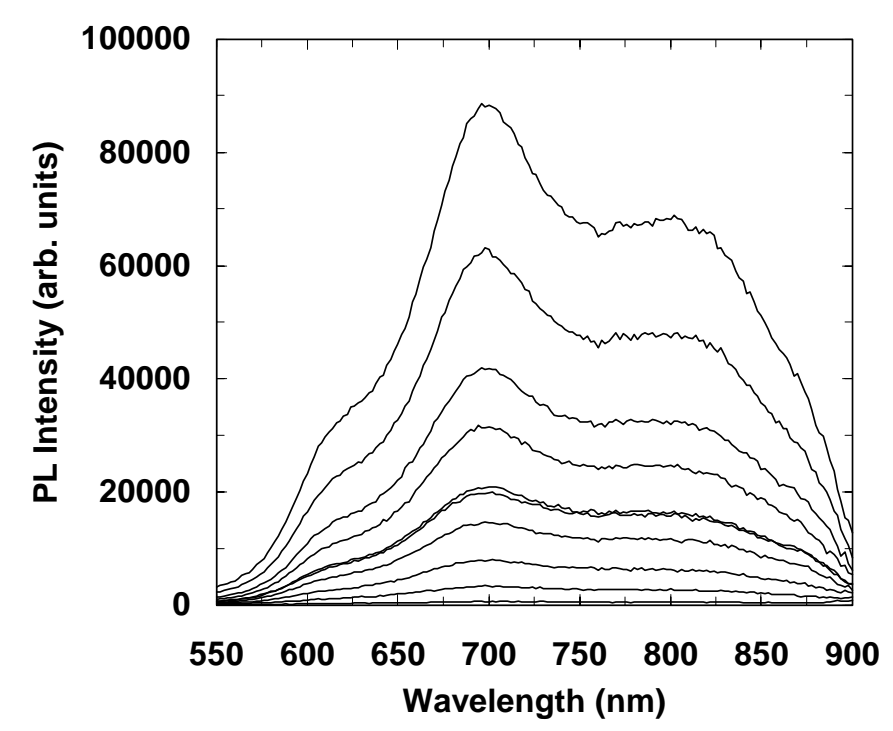

Figure 3.PL spectra of a-SiN $\mathrm{N}_{\mathrm{x}} \mathrm{H}$ in the $0.1-3.7 \mathrm{~W} \mathrm{~cm}^{-2}$ laser intensity range at $12 \mathrm{~K}$.

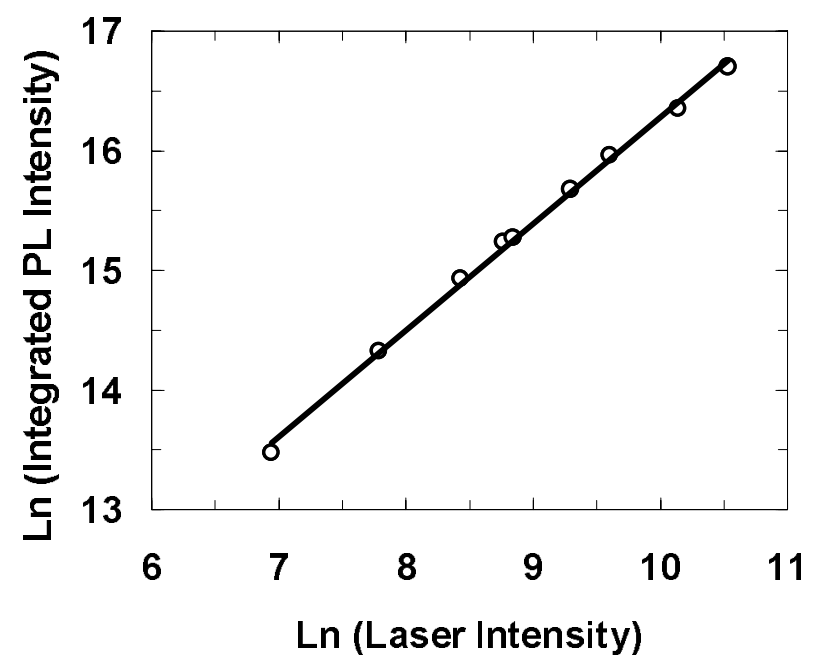

Figure 4. Dependence of a-SiN $\mathrm{x}: \mathrm{H}$ integrated PL intensity versus excitation laser intensity at $12 \mathrm{~K}$.

\subsection{Surface morphology of the amorphous silicon}

Atomic force microscopy (AFM) has been performed the on the a-SiN $\mathrm{x}: \mathrm{H}$ samples to characterize the morphology of the surface and to analyze origin of the luminescence. The surface of the $\mathrm{a}-\mathrm{SiN}_{\mathrm{x}}: \mathrm{H}$ is optically flat and thus amenable for the growth of multiple layers. This is necessary condition for the realization of the one-dimensional PBG microcavity. The surface morphology of the a-SiNx:H is quite uniform. The bulk of the material is composed of globules of a-SiNx:H and is similar to $\pi$-Si. Figure 5 shows the medium resolution AFM picture of the silicon rich aSiNx:H surface. Additionally, although the surface is flat optically, it is quite rough in the $100 \mathrm{~nm}$ range, which corresponds to the average globule size. 


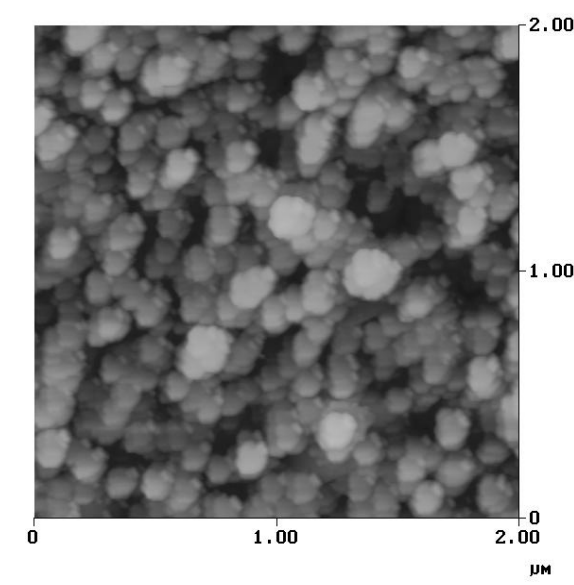

Figure 5. Medium resolution AFM of the silicon rich a-SiNx:H surface.

\section{PHOTONIC MICROCAVITY}

An optical resonator, the optical counterpart of an electronic resonant circuit, confines and stores light at certain resonance frequencies. These specific frequencies correspond to the optical modes of the microcavity. The microcavity may be viewed as an optical transmission system incorporating feedback. Light circulates or is repeatedly reflected within the system. Using the right geometry it is possible to make one-, two-, or three dimensional optical resonators. Planar and spherical mirror resonators, ring resonators, and optical fiber resonators are the most typical optical resonators. The simplest optical resonator is the planar mirror resonator, often called the Fabry-Perot resonator, which comprises two parallel planar mirrors between which light is repeatedly reflected with little loss. It was introduced by A. Fabry and Ch. Perot in 1899. The initial optical resonator was fabricated using two partially coated mirrors. Later on dielectric and semiconductor materials were also used, since the Fresnel reflections at high refractive index step at the material-air interface produces natural mirrors. The first instrumental passive optical resonator was developed by Charles Fabry and Alfred Perot, and is now more than a century old. The original paper on the interference spectroscope was published in the Annales de Chimie et de Physique in $1899^{18}$.

\subsection{Cavity Quantum Electrodynamics}

In a microcavity, the photon density of states (DOS) are enhanced at the cavity resonances, when compared with the continuum of photon DOS of a bulk sample. The spontaneous emission (SE) cross-sections at the microcavity resonances are larger than the bulk SE cross-sections because of the enhanced photon DOS. Also, the luminescence cross-sections in between the microcavity resonances are smaller than the bulk SE cross-sections. The cavity alteration of the SE was first proposed for radio waves. ${ }^{19}$ The possibility to control the emission properties of materials with the use of optical microcavities ${ }^{20}$ attracted the attention of the photonics community. ${ }^{21}$ Afterwards, the microcavity alteration of the spontaneous emission was proposed ${ }^{22}$ and observed in organic microcavities, ${ }^{23}$ in the optical part of the electromagnetic spectrum. ${ }^{24}$ In addition, alteration of the SE in semiconductor microcavities was observed ${ }^{25}$ and calculated. ${ }^{26}$ In this weak coupling (of the photon and exciton modes) regime, the interaction (Rabi coupling) strength is smaller than the microcavity mode damping (linewidth) and the exciton mode damping (linewidth). The SE spectrum is altered due to a redistribution of the DOS by the presence of the microcavity. However, in the strong coupling (of the photon and exciton modes) regime, the interaction (Rabi coupling) strength is bigger than the microcavity mode damping (linewidth) and exciton mode damping (linewidth), and Rabi splitting of the microcavity and exciton modes occurs. ${ }^{27}$ The proposition of strong coupling in optical microcavities, ${ }^{28}$ was followed by its observation ${ }^{29}$ and theory. ${ }^{30}$ If a cavity mode is probed out of the cavity, a resonant change of SE light intensity must be detected. This effect corresponding to a light intensity spatial redistribution is observed even in the absence of any sizeable resonant change of the average SE rate. ${ }^{31}$ Since the lifetime changes are the most essential effect in the original concept of the modification of SE proposed by Purcell. ${ }^{32}$ Much attention has been paid to the observation of the cavity induced changes of the SE decay rate. ${ }^{33}$ However, the rate of change of SE lifetime in one dimensionally confined microcavity structures has been predicted theoretically to be relatively small. ${ }^{34}$ 


\subsection{Photonic Bandgap Materials}

With the introduction of photonic bandgap (PBG) concept in 1987 independently by both Eli Yablonovitch ${ }^{35}$ and Sajev John ${ }^{36}$ suddenly other ways to fabricate microcavities appeared. PBG materials have indeed attracted much attention from the optics and optoelectronics community ${ }^{37}$. PBG materials are constructed by spatially patterning the dielectric function of a medium in a periodic way. A medium engineered in such a way exhibits photonic stop bands, i.e., PBG's, for a certain range of frequencies of the electromagnetic spectrum. A PBG structure can also be treated as a photonic crystal constructed from "photonic atoms", i.e., dielectric "photonic resonators", which possess electromagnetic resonances at discrete frequencies. Photonic atoms are the electromagnetic analogue of the real "electronic" atoms, which possess electronic resonances at discrete frequencies.

\subsection{Distributed Bragg Reflector}

The distributed Bragg reflector (DBR) is the simplest one-dimensional PBG. If electromagnetic radiation falls onto a structure consisting of thin films of several different materials, multiple reflections will take place within the structure. Exact analysis for the electromagnetic wave propagation in such structures can be done by the transfer matrix method (TMM) ${ }^{38}$ The simplest nontrivial periodic multilayer system to analyze is the one, whose period consists of two layers of differing refractive indices $\mathrm{n}_{1}, \mathrm{n}_{2}$ both having the same optical thickness. Such a system has a reflectance maximum at a wavelength, for which the optical thickness is a quarter wavelength and is called a simple quarter-wave stack or a DBR.

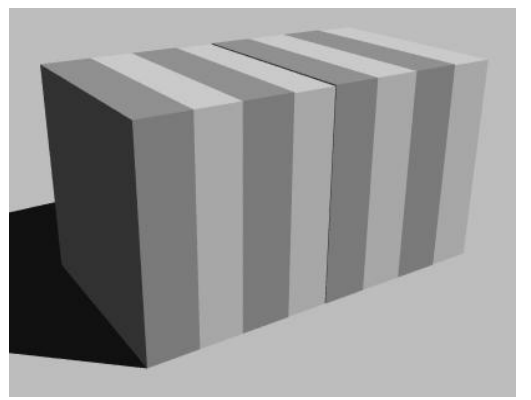

Figure 6. Schematic of an 8 layer distributed Bragg reflector (DBR)

The reflectance of a number of a-SiO $\mathrm{x}: \mathrm{H}$ and a-SiN $\mathrm{x}: \mathrm{H}$ quarter wave stacks, which are used as mirrors of the FabryPerot cavity, calculated by TMM is shown in figure 7 as a function of the (normalized) reciprocal wavelength. Figure 7 illustrates that, as the number $(\mathrm{N})$ of periods in the stack increases, the reflectance at $\lambda_{\mathrm{o}}$ approaches unity. The transition from reflection to transmission becomes sharper, and the number of oscillations outside the rejection band increases. As $\mathrm{N}$ approaches infinity, the reflectance approaches unity over a band, i.e., the high reflectance zone. For $\mathrm{N}=7, \mathrm{~N}=10$ and $\mathrm{N}=14$, the reflectance maximum is 91,97 , and $99 \%$, respectively. The ratio $\mathrm{n}_{\mathrm{L}} / \mathrm{n}_{\mathrm{H}}$ controls both the width and the height of the of the reflectance band.

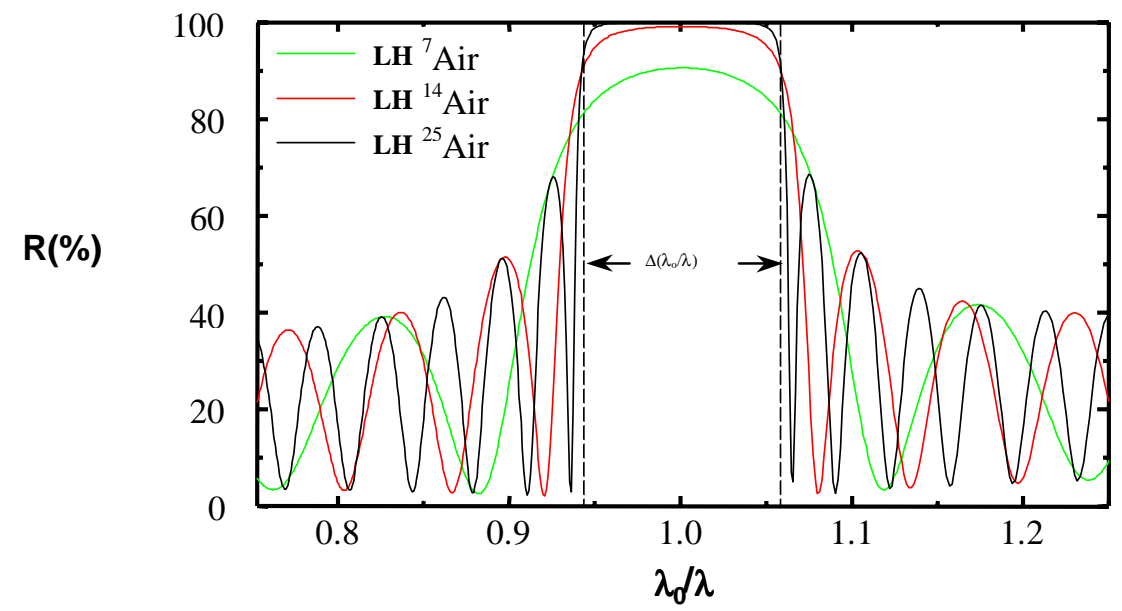

Figure 7. Calculated normalized reflectance spectra of 7, 14, 25 quarter-wave stack DBR's. L and H stand for low $\left(\mathrm{a}-\mathrm{SiO}_{\mathrm{x}}: \mathrm{H}\right)$ and high $\left(\mathrm{a}-\mathrm{SiN}_{\mathrm{x}}: \mathrm{H}\right)$ refractive index layers of quarter wave thickness at $710 \mathrm{~nm}$. 


\subsection{The DBR Microcavity Analysis}



Figure 8. Calculated reflectance curves of microcavities with 7, 10, and 14 pairs of DBR layers on both sides L and $\mathrm{H}$ stand for low and high refractive index layers of quarter wave thickness at $710 \mathrm{~nm}$.

The response of a number of microcavities with different number of periods in the quarter wave stack is shown in figure 8. The calculations are performed by the transfer matrix method (TMM). Figure 8 illustrates that, as the number of periods in the stack increases, the reflectance at $\lambda_{\mathrm{o}}$ approaches unity. The transition from reflection to transmission becomes sharper, and the number of oscillations outside the rejection band increases. For $\mathrm{N}=7, \mathrm{~N}=10$ and $\mathrm{N}=14$, the reflectance maximum is 91,97 , and $99 \%$, respectively. The ratio $\mathrm{n}_{\mathrm{L}} / \mathrm{n}_{\mathrm{H}}$ controls both the width and the height of the of the reflectance band. Additionally, as the reflectance of the mirrors increases, the resonant transmission bandwidth at the center design wavelength of $710 \mathrm{~nm}$ decreases. The decrease of the resonant transmission bandwidth results in high quality factor resonances.

\subsection{Dielectric Microcavity fabricated without ammonia}
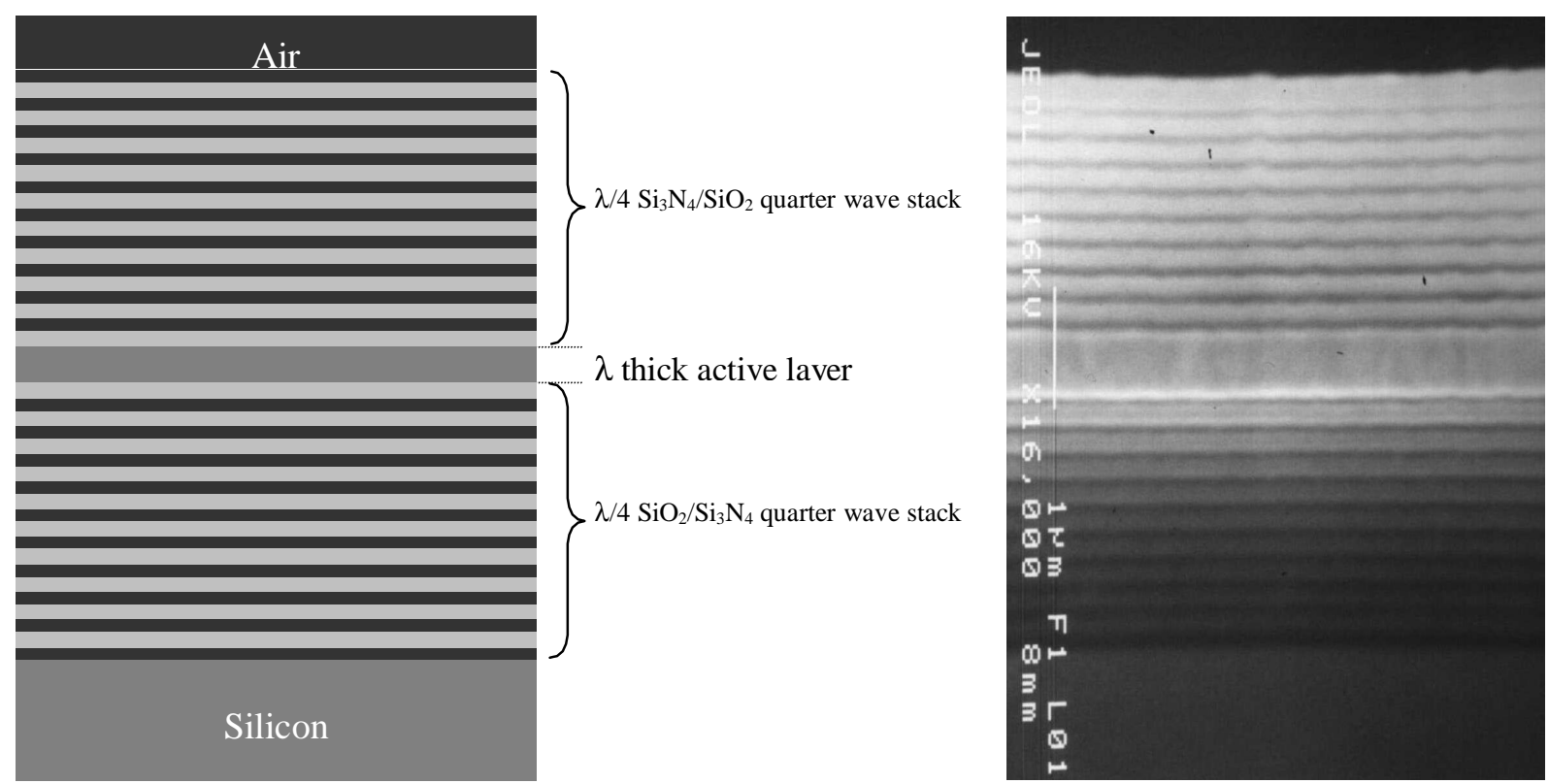

Figure 9. The planar microcavity structure with DBR mirrors. 
The schematic and the scanning electron microscope (SEM) picture of one of the microcavity structures is shown in figure 9. The $328 \mathrm{~nm}$ thick a-SiN : $\mathrm{H}$ layer can be distinguished in the middle, although it is mixed up a little with the silicon oxide layers enclosing it. The dark regions are $104 \mathrm{~nm}$ thick silicon nitride layers, and the light gray regions are $123 \mathrm{~nm}$ thick silicon oxide layers. The dielectric microcavity was realized by a $\lambda / 2$ active layer of a-SiN $: \mathrm{H}_{\mathrm{sandwiched}}$ between two passive DBR's. First, the bottom DBR was deposited by PECVD on the silicon substrate using $\lambda / 4$ alternating layers of $\mathrm{a}-\mathrm{SiN}_{\mathrm{x}}: \mathrm{H}$ and $\mathrm{a}-\mathrm{SiO}_{\mathrm{x}}: \mathrm{H}$. For the nitrogen rich a-SiN $\mathrm{x}: \mathrm{H}$ deposition, ammonia $\left(\mathrm{NH}_{3}\right)$ with a flow rate of $10 \mathrm{sccm}$, and $2 \%$ silane $\left(\mathrm{SiH}_{4}\right)$ in nitrogen $\left(\mathrm{N}_{2}\right)$ with a flow rate of $180 \mathrm{sccm}$ were used. For the a-SiO : $\mathrm{H}$ deposition, nitrous oxide $\left(\mathrm{N}_{2} \mathrm{O}\right)$ with a flow rate of $25 \mathrm{sccm}$, and $2 \% \mathrm{SiH}_{4}$ in $\mathrm{N}_{2}$ with a flow rate of $180 \mathrm{sccm}$ were used. After the deposition of the bottom DBR, a $\lambda / 2$ layer of silicon rich a-SiN $\mathrm{x}_{\mathrm{x}} \mathrm{H}$ was deposited. For the silicon rich a-SiN $\mathrm{N}$ deposition, only $2 \% \mathrm{SiH}_{4}$ in $\mathrm{N}_{2}$ with a flow rate of $180 \mathrm{sccm}$ was used. Afterwards, the top DBR was deposited, using $\lambda / 4$ alternating layers of $\mathrm{a}-\mathrm{SiO}_{\mathrm{x}}: \mathrm{H}$ and $\mathrm{a}-\mathrm{SiN}_{\mathrm{x}}: \mathrm{H}$. The $\mathrm{RF}$ power was $20 \mathrm{~W}$, and the deposition chamber pressure $1 \mathrm{Torr}$ during the continuous deposition process. The PL spectrum of bulk amorphous silicon has an maximum emission at 710 $\mathrm{nm}$. This yields geometrical layer thicknesses of $164 \mathrm{~nm}$ for the half wavelength hydrogenated amorphous silicon active layers, respectively, and $123 \mathrm{~nm}$ and $104 \mathrm{~nm}$ for the silicon oxide and silicon nitride DBR layers, respectively. The reflectance spectra of a DBR is shown in figure 10. The DBR is highly reflective over a stop band range of $80 \mathrm{~nm}$. Figure 11 shows the reflectance spectra of the half wavelength microcavity.

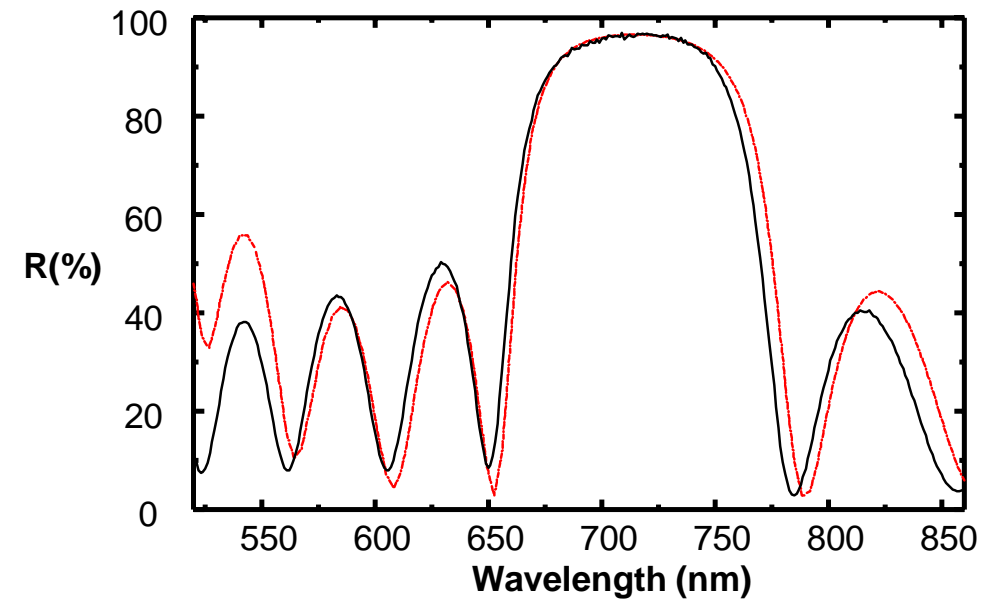

Figure 10. Experimental (solid line) and calculated (dotted line) reflectance spectra of a DBR.

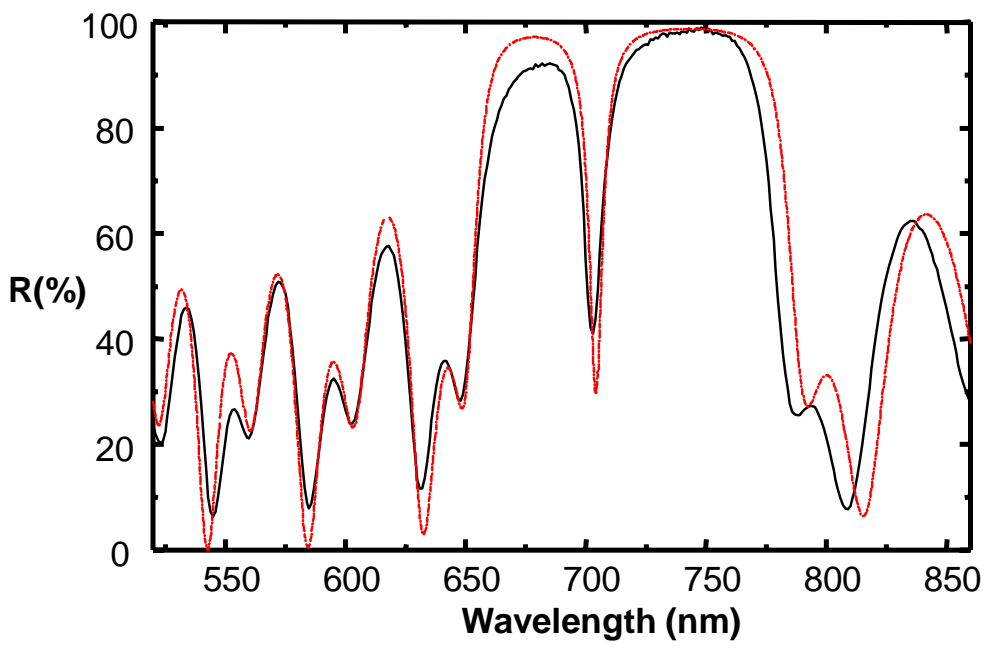

Figure 11. Experimental (solid line) and calculated (dotted line) reflectance spectra of half wavelength thick microcavity formed by 2 DBR mirrors. 
Reflectance vs. wavelength and frequency spectrum of a half wavelength thick microcavity enclosed between two DBR's, which have alternating a-SiN $\mathrm{x}: \mathrm{H}$ and a-SiO $\mathrm{x}: \mathrm{H}$ layers is shown in figure 11. In order to clarify the effect of the microcavity and to demonstrate its advantages with respect to the bulk Si rich a-SiNx:H, we also show the PL of a $\lambda / 2$ thick layer of bulk $\mathrm{Si}$ rich a-SiNx:H in figure 12. Both spectra are obtained under the same experimental conditions. The red-near-infrared PL of the bulk Si rich a-SiNx:H has a broad linewidth of $250 \mathrm{~nm}$. This broad linewidth shows that, $\mathrm{Si}$ rich a-SiNx:H has potential as a novel photonic gain medium. A comparison of the spectra in the previous figures shows that the effect of the microcavity is twofold: first, the wide emission band $(250 \mathrm{~nm})$ is strongly narrowed to $6 \mathrm{~nm}$; second, the resonant enhancement of the peak PL intensity is more than one order of magnitude with respect to the emission of the $\lambda / 2$ thick layer of bulk $\mathrm{Si}$ rich a-SiNx:H (assuming that all the transmitted excitation light is pumping the active layer in both cases, with a transmittance of approximately $70 \%$ at the excitation wavelength for both cases). In addition by choosing the appropriate width of the Si rich a-SiNx:H active layer and DBR's, it is possible to select the emission wavelength of the microcavity by taking advantage of the broad spectral emission of the a-SiNx:H active layer. Both spectra are plotted together in figure 12.

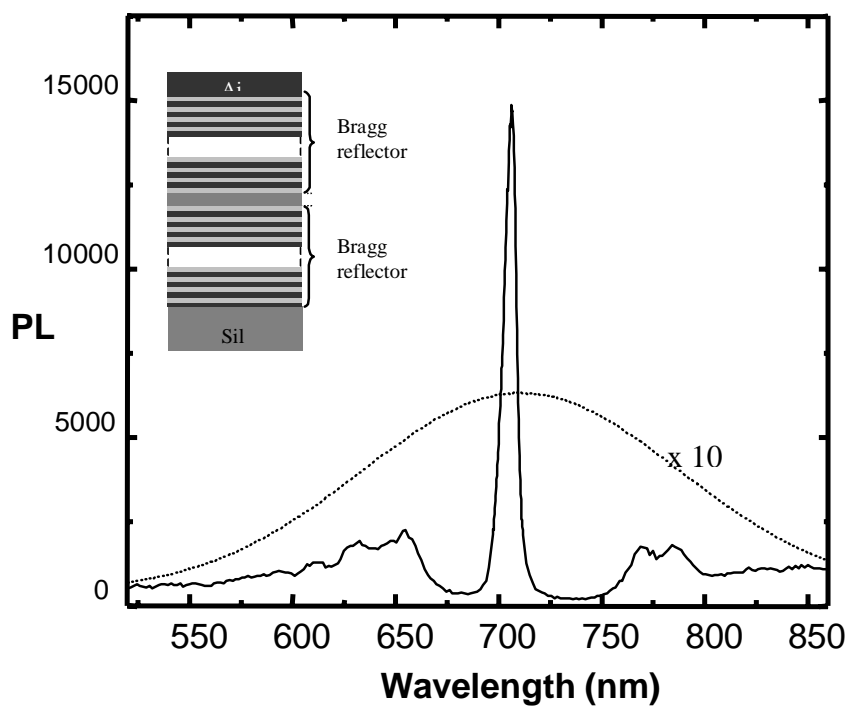

Figure 12. PL spectrum of a half wavelength thick microcavity with 2 DBR mirrors (solid line) and bulk amorphous silicon (dotted line). The data of the amorphous silicon were multiplied by 10 for comparison.

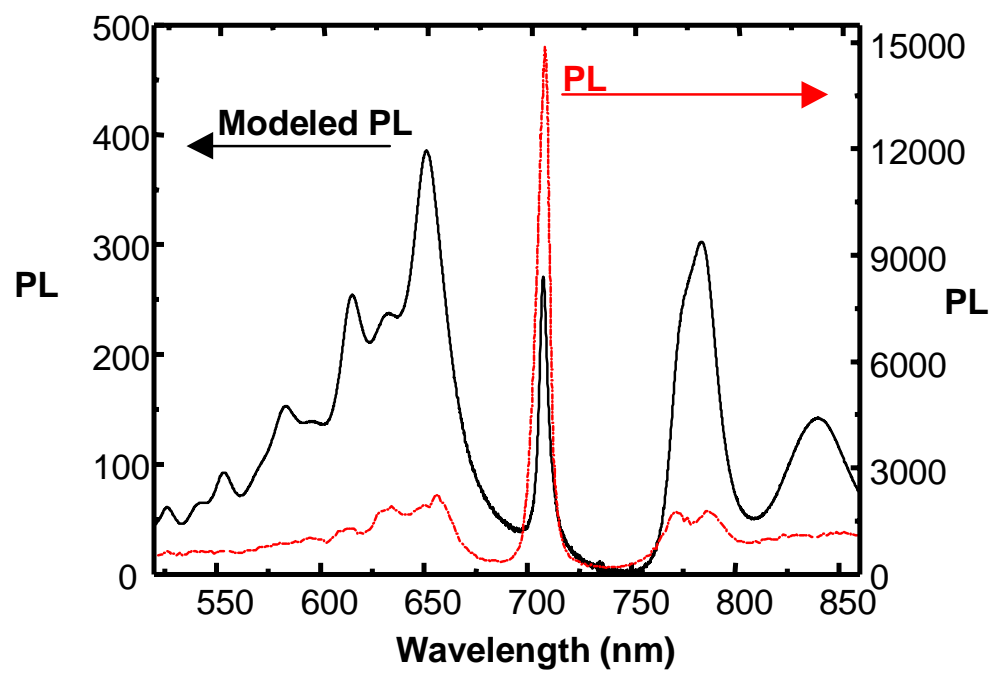

Figure 13. Modeled emission spectra (solid line) and PL (dotted line) of a half wavelength thick dielectric microcavity. 
In order to prove that the microcavity structure does not act to simply filter the SE, which would yield only a relative and not an absolute enhancement the emission spectrum of a half wavelength thick microcavity was modeled as the PL spectrum of the bulk amorphous silicon multiplied by the transmission spectrum of the sample. The spectral shape of PL can not be reproduced by simply multiplying the PL spectrum of the bulk by the transmittance of the sample as shown in figure 13

\section{CONCLUSIONS}

Bulk and microcavity luminescence properties of hydrogenated amorphous silicon nitride (a-SiN $: \mathrm{H})$ were studied at room temperature and at low temperatures. The PL emission intensity increases at low temperatures due to the lack of thermally excited phonons. The emission spectrum stays broad even at low temperatures. The broad luminescence spectrum even at low temperatures is a results of the heterogenous size distribution of the amorphous silicon quantum dots. This heterogenous size distribution is further confirmed by atomic force microscopy (AFM) measurements of the sample surface. The broad PL spectrum of the a-SiN $\mathrm{S}_{\mathrm{x}} \mathrm{H}$, also makes it a suitable source for wavelength division multiplexing (WDM) applications. Dielectric Fabry-Perot microcavities are used for the enhancement and inhibition of $\mathrm{PL}$ in a-SiN $\mathrm{N}_{\mathrm{x}}: \mathrm{H}$. The microcavity mode was tuned to the emission maximum of a-SiN$: \mathrm{H}(710 \mathrm{~nm})$. The reflectance and the PL measurements show the effect of the microcavity. The a-SiN $: \mathrm{H}$ microcavities consist of an active $(\mathrm{a}-\mathrm{SiN}$ : $\mathrm{H})$ layer sandwiched between two mirrors. Dielectric microcavities were realized using distributed Bragg reflectors (DBR's). This is the same structure used in vertical cavity surface emitting lasers (VCSEL's). Stopband widths of 80 $\mathrm{nm}$ and $99 \%$ reflection at the middle of the stopbands were obtained by using alternating quarter wavelength thick a$\mathrm{SiO}_{\mathrm{x}}: \mathrm{H}$ and a-SiN $\mathrm{x}: \mathrm{H}$ layers. The DBR's were fabricated using alternating layers of quarter wavelength thick a-SiN $\mathrm{N}_{\mathrm{x}}: \mathrm{H}$ and $\mathrm{a}-\mathrm{SiO}_{\mathrm{x}}: \mathrm{H}$. The reflectance spectra were simulated by the transfer matrix method (TMM). Excellent agreement between the theoretical and experimental spectra was obtained. The effect of the number of top mirror layer pairs on the emission maximum and the transmittance at the microcavity resonance was investigated both theoretically and experimentally. The reflectance of the DBR increases as the number of layer pairs in the dielectric stack increases. This, in turn, reduces the emission maximum and the transmittance at the cavity resonance, because of the reduced light coupling out of the microcavity. The PL of the bulk a-SiN $: \mathrm{H}$ is enhanced at, and inhibited in between, the resonances of the microcavity, with respect to the bulk a-SiN $\mathrm{x}: \mathrm{H}$. The enhancement and inhibition of the PL is understood by the modified photon density of states (DOS) of the microcavity. The PL bandwidth was narrowed to a linewidth of $6 \mathrm{~nm}$, with respect to the linewidth of the bulk a-SiN $\mathrm{x}: \mathrm{H}$, again due to the presence of the electromagnetic modes of the microcavity. Quality factors as high as 120 was obtained. This narrowing and enhancement of the PL can be understood by the redistribution of the density of optical modes due to the presence of the microcavity. The microcavity enhancement and inhibition of luminescence in a-SiN $\mathrm{x}_{\mathrm{x}} \mathrm{H}$ opens up a variety of possibilities for optoelectronic applications such as color flat panel displays or resonant cavity enhanced (RCE) devices for WDM applications.

\section{ACKNOWLEDGMENTS}

We would like to acknowledge the partial support of this research by the Scientific and Technical Research Council of Turkey (TUBITAK) Grant No: TBAG-1952 and the European Office of Aerospace Research and Development (EOARD) Grants No: F61775-01-WE062 and FA8655-02-M-4086.

\section{REFERENCES}

1 J.D. Joannopoulos and G. Lucovsky, "The physics of hydrogenated amorphous silicon," (Springer Verlag, Berlin, 1984).

2 S. Perkowitz, "Optical Characterization of Semiconductors," (Academic Press, London, 1993), p.27.

3 P. Shaoqi and D. Xiaoning, "Properties of Sputtered Amorphous Silicon without Hydrogen", in "Proceedings of the International Workshop on Amorphous Semiconductors," H. Fritzsche, D. Han, C. C. Tsai, Eds. (World Scientific, Singapore, 1987).

4 J. I. Panvoke, "Luminescence in Hydrogenated Amorphous Silicon", in "Proceedings of the International Workshop on Amorphous Semiconductors," H. Fritzsche, D. Han, C. C. Tsai, Eds., (World Scientific, Singapore, 1987).

5 Y. Yamamoto and R. E. Slusher, "Optical Processes in Microcavities," Phys. Today, 46, 66 (1993).

6 R. E. Slusher and C. Weisbuch, "Optical Microcavities in Condensed Matter Systems," Solid State. Commun., 92, 149 (1994).

7 H. Zimmermann, "Integrated Silicon Optoelectronics," (Springer Verlag, Berlin, 2000) pp. 1-352. 
8 R. A. Street, Ed., "Technology and Applications of Amorphous Silicon,” (Springer Verlag, Berlin, 2000), pp. 1-403.

9 A. M. Agarwal, L. Liao, J. S. Foresi, M. R. Black, X. Duan, and L.C. Kimerling, "Low-loss Polycrystalline Silicon Waveguides for Silicon Photonics," J. Appl. Phys. 80, 6120 (1996).

10 B. Jalali, S. Yegnanarayanan, T. Yoon, T. Yoshimoto, I. Rendina, and F. Coppinger, "Advances in Silicon on insulator Optoelectronics," IEEE J. Select. Topics in Quantum Electron. 4, 938 (1998).

11 A. Kaneko, T. Goh, H. Yamada, T. Tanaka, and I. Ogawa "Design and applications of silica-based planar lightwave circuits," IEEE J. Select. Topics in Quantum Electron. 5, 1227 (1999).

12 B. J. Offrein, R. Germann, F. Horst, H.W.M. Salemink, R. Beyerl, and G. L. Bona, "Resonant coupler-based tunable add-afterdrop filter in silicon-oxynitride technology for WDM networks," IEEE J. Select. Topics in Quantum Electron. 5, 1400 (1999).

13 L. Pavesi, R. Guardini, and C. Mazolleni, "Porous Silicon Resonant Cavity Light Emitting Diodes," Solid State Commun. 97, 1051 (1996).

14 B. T. Sullivan, D. J. Lockwood, H. J. Labbe, and Z.-H. Lu, "Photoluminescence in Amorphous Si/SiOx Superlattices Fabricated by Magnetron Sputtering," Appl. Phys. Lett. 69, 3149 (1996).

15 A. Serpengüzel, A. Aydinli, and A. Bek, "Enhancement and inhibition of photoluminescence in hydrogenated amorphous silicon nitride microcavities, " Optics Express 1, 108 (1997).

16 M. H. Brodsky, "Quantum Well Model of the Hydrogenated Amorphous Silicon," Solid State Commun. 36, 55 - 59 (1980).

17 A. Serpengüzel, A. Aydinli, A. Bek, and M. Güre, "Visible photoluminescence from planar amorphous silicon nitride microcavities, " J. Opt. Soc. Am. B. 15, 2706 (1998).

18 Ch. Fabry and A. Perot, "Theorie et applications d'une nouvelle methode de spectroscopie interferentielle," Ann. Chim. Phys. 16, 115-144 (1899).

19 E. M. Purcell, "Spontaneous Emission Probabilities at Radio Frequencies," Physical Review 69, 681 (1946).

20 K. H. Drexhage, "Progress in Optics,” Vol. 12, E. Wolf, Ed., (North-Holland, New York, 1974), p. 165.

21 J. Rarity and C. Weisbuch, Eds., "Microcavities and Photonic Bandgaps: Physics and Applications," (Kluver, Dordrecht, 1996).

22 P. W. Milloni and P. L. Knight, "Spontaneous emission between mirrors," Opt. Commun. 9, 119 - 122 (1973).

23 F. De Martini, G. Innocenti, G. R. Jacobowitz, and P. Mataloni, "Anomalous Spontaneous Emission Time in a Microscopic Optical Cavity," Phys. Rev. Lett. 59, 2955 - 2958 (1987).

24 Y. Yamamoto, S. Machida, K. Igeta, G. Bjork, "Controlled Spontaneous Emission in Microcavity Semiconductor Lasers", in Y. Yamamoto, Ed., "Coherence, Amplification, and Quantum Effects in Semiconductor Lasers," Series in Pure and Applied Optics (Wiley, New York, 1991).

25 H. Yokoyama, K. Nishi, T. Anan, H. Yamada, S. D. Brorson, and E. P. Ippen, "Enhanced Spontaneous Emission from GaAs quantum Wells in Monolithic Microcavities," Appl. Phys. Lett. 57, 2814 - 2816 (1990).

26 G. Björk, S. Machida, Y. Yamamoto, and K. Igeta, "Modification of spontaneous emission rate in planar dielectric microstructures, Physical Review A 44, 669 - 681 (1991).

27 M. S. Skolnick, T. A. Fisher, and D. M. Whittaker, "Strong Coupling Phenomena in Quantum Microcavity Structures," Semiconductor Science and Technology 13, 645 - 669 (1998).

28 Y. Zhu, J. Gauthier, S. E. Morin, Q. Wu, H.J. Carmichael, and T.W. Mossberg, "Vacuum Rabi splitting as a feature of linear dispersion theory: analysis and experimental observations," Phys Rev. Lett. 64, 2499 - 2502 (1990).

29 C. Weisbuch, M. Nishioka, A. Ishikawa, and Y. Arakawa, "Observation of coupled exciton-photon mode splitting in a semiconductor quantum microcavity," Phys. Rev. Lett. 69, 3314 - 3317 (1992).

30 S. Pau, G. Björk, J. Jacobson, H. Cao, and Y. Yamamoto, "Microcavity exciton-polariton splitting in the linear regime," Physical Review B 51, 14437 - 14447 (1995).

31 F. De Martini, G. Innocenti, G.R Jacobovitz and P. Mataloni, "Anomalous Spontaneous Emission Time in a Microscopic Optical Cavity", Physical Review Letters 59, 2955 (1987).

32 E. M. Purcell, "Spontaneous Emission Probabilities at Radio Frequencies", Physical Review 69, 681 (1946).

33 K. Tanaka, T. Nakamura, W. Takamatsu, M. Yamanishi, Y. Lee and T. Ishihara, Cavity Induced Changes of Spontaneous Emission Lifetime in One-Dimensional Semiconductor Microcavities", Physical Review Letters 74, 3380 (1995).

34 Y. Yamamoto, S. Machida, and G. Bjork, "Microcavity Semiconductor Laser with Enhanced Spontaneous Emission", Physical Review A 44, 657 (1991).

35 E. Yablonovitch, "Inhibited spontaneous emission in solid-state physics and electronics," Phys. Rev. Lett. 58, 2059-2062, 1987.

36 S. John, "Strong localization of photons in certain disordered dielectric superlattices," Phys. Rev. Lett. 58, $2486-2489,1987$.

37 J. D. Joannopoulos, R. D. Meade, and J. N. Winn, "Photonic Crystals," (Princeton University Press, New Jersey, 1995) pp. 1129.

38 L. Levi, “Applied Optics: A Guide to Optical System Design,” Vol. 2, (Wiley, New York, 1980) pp. 47-67. 